before his attendance upon labours were limited to the management of normal pregnancy, labour, and the puerperium, together with such complications as are common. Instruction of this kind could well be given by the demonstrator and would clear the ground for the lecturer on obstetrics, leaving him free to devote more time to the graver complications and more advanced work. The student also at this later stage of his career would be in an infinitely better position to appreciate the value of this advanced teaching.

Another method of instruction, and one likely to be keenly appreciated by advanced students, is the delivery of clinical lectures upon cases recently in the wards. Hitherto, owing to the absence of lying-in wards, such lectures have seldom been delivered. Amongst the many able and learned obstetricians in London who have from time to time endeavoured to raise the standard of teaching there is probably no one to whom we owe so much as to the late Dr. Matthews Duncan. He came to London to fill the vacant post of obstetric physician at St. Bartholomew's Hospital in October, 1877, and from the first devoted a large portion of his time and his great experience, and gradually his affection also, to his wards and his stadents. A "Scotsman of the Scots," he came to London believing that there was little of value to be found South of the Tweed. He gradually transferred his devotion from Edinburgh and Aberdeen to London and St. Bartholomew's. He would go to Scotland for his holiday but came back to London for his work. His influence at the hospital may be summed up in the statement that by his teaching and example be transformed the teaching of obstetrics and gynæcology from an almost insignificant position to one of the greatest importance. This change was soon felt, not only by his colleagues on the staff of St. Bartholomew's but by the strdents and junior officers who flocked to his lectures and wards to learn from his example that the practice of midwifery and gynæcology could be as noble and as full of dignity as that of the highest standards of surgery and medicine. This transformation was due not only to his learning and greatness as a teacher and to his success as a leading consultant--both of which were sufficiently recognised far beyond the limits of St. Bartholomew's and even of London itself-but also to the greatness of character which made it impossible for anyone to be brought in contact with him, either in hospital or palace, without feeling that the branch of the profession which he practised was as noble and dignified as that practised by the greatest physicians and surgeons. In this respect, as in the high standard of his teaching, his example diffused itself in every direction, and it is no injustice to other great teachers of his time to say that it raised the whole standard of teaching obstetrics and gynæcology throughout London. It is difficult to say whether he excelled most in his systematic or clinical lectures. Students who regularly attended his systematic lectures received a course of instruction which by its completeness rarely left any matter of importance-however recent a contribution or emanating from whatever countrylacking in their knowledge of the subject. Illumined with the criticism of his clear, highly-trained, logical mind, they were enabled to discriminate what was of permanent value and what they might expect to lay aside with fuller knowledge. His clinical lectures were models of what such lectures should be. No student could help keeping his attention fixed on the subject. No mass of detail was allowed to obscure the clearness of the main object of the lecture, so that every one carried away some important principle clearly impressed on his mind, while to the more advanced student in gynæcology the facts grouped round the main thesis (the importance of which was not always perceptible to the elementary student) afforded him further instruction, the value of which rose in proportion to his knowledge of the subject and his experience as a practitioner. The influence of such a man who combined the highest personal qualities with knowledge, experience, and clear judgment, not only in the ward and classroom but in every relation of life, raised the standard of the science and practice of obstetrics and gynæcology to a position which, at least in London, they had never before attained.

The Stwage of Tavistock.-At a special meeting of the Tavistock (Devon) urban council held on August 15th a tender was accepted for $£ 4544$ for carrying out a sewage disposal scheme for the town.

\section{SOME OBSERVATIONS ON THE EFFECT OF STERILE CASEOUS MATTER IN THE TREATMENT OF TUBER- CULOUS DISEASE.}

By PETER PATERSON, M.B., O.M.GLasG., M.R.C.S.ENG., L.R.C.P. LOND.,

SURGEON TO THE ROYAL INFIRMARY, GLASGOW.

I WISH to direct attention in this preliminary paper to a few observations which $I$ have made on the effect produced in tuberculous lesions by the injection of some of the products of the tubercle bacillus as elaborated in the body.

It is well known that tubercle bacilli can easily be found in the walls of tuberculous abscesses but in the contents they may be so few that inoculation experiments may be necessary to demonstrate their presence. The conditions in the interior of such abscesses are apparently favourable to growth; the serum and soluble toxins pass into the circulation, producing more or less constitutional disturbance, whilst there is a stream of fresh serum, rich in nutritive substances, into the interior; the temperature is most suitable, and still not only do the bacilli not increase but actually diminish in numbers. There must, then, be some substance in the contents which is inimical to the bacilli and, as the fluids are being constantly renewed, presumably this deleterious material is in the encapsulated solids.

The conditions which exist in an abscess, as regards the relative proportion of caseous matter, cannot be produced in the body as a whole, still the possibility remained that the introduction of small quantities might exert a beneficial influence on the disease. But before such injections could be made it was necessary to destroy the living bacilli present, for though these may be so few as to escape notice with the microscope, inoculations show that even in these cases they are present. As it was requisite that the chemical constitution of the solids should be as little changed as possible it was evident that neither heat nor chemicals could be used for the purpose of sterilisation, and extreme cold, even though applied continuously for a long time, does not affect the virulence of organisms. Cold applied intermittently was found to produce the desired result. If tuberculous pus be kept in a refrigerator for five or six months, but allowing it to thaw frequently during that time, the pus will not induce tuberculosis when injected into guinea-pigs, even in large doses. Having destroyed the bacilli by this means the serum is poured off and the sediment is then washed for three days in a continuous stream of cold sterilised water to remove all soluble toxins. Sterile normal salt solution is now added to the fine white sediment till each cubic centimetre of the emulsion contains five milligrammes of solid substance and the material is then ready for use. Large numbers of animals have been injected with this suspension and in no instance has tuberculosis been produced. If a large quantity be injected into a tuberculous patient there is a well-marked reaction but a few minims can be given without any evident disturbance. If the opsonic index is to be regarded as the degree of resistance of the body against any particular organism then we have in this substance a means of raising the tuberculous index to a high level and that without any negative phase. In one of my patients this index rose from 0.5 to normal limits within 12 hours. Such a rapid rise is unusual but there is no difficulty in raising the index to what is regarded as normal and in maintaining it at that level. The technique necessary to obtain this index is so. elaborate that it can only be conducted in a fully equipped laboratory and by a properly trained observer, and so lcng as it remains in this position it can only be employed in a limited number of cases. In using caseous matter I have found the temperature a convenient guide to the dose. If sufficient be introduced to raise the temperature from $0.5^{\circ}$ to $1^{\circ}$ the best results are obtained and at the same time the index keeps within normal limits. If there be no rise of temperature the index may still be high and yet little, if any, improvement be observed in the disease. I have found that from 0.1 to 0.5 cubic centimetre of emulsion given every second day is sufficient to raise both index and temperature. I begin with the smaller quantity and increase the amount at each injection till a slight reaction appears. The following 
is a short summary of several cases in which this treatment has been tried.

CASE 1.-The patient was a girl, aged eight years, with old-standing hip-joint disease. The joint was ankylosed and a sinus led from the outer side of the thigh up towards the joint. The skin round the opening of the sinus was extensively and deeply ulcerated. The sinus closed and the nlcers healed with four weeks' treatment.

CASE 2.-The patient, a boy, aged nine years, had tuberculous disease of the left clavicle, the left tarsus, and the right fibula. The left foot had been amputated and the fibula destroyed for about five inches before the treatment was commenced. When the injections were started there was a long open wound over the fibula and a sinus, discharging pus, over the clavicle. These were healed within five weeks after the injections were commenced.

CASE 3.-The patient was a girl, aged 14 years, with disease of the synovial membranes of the wrist-joint which was discharging through two sinuses. After seven weeks' treatment the swelling had disappeared and the sinuses closed. The movements, though improved, are still restricted.

CASE 4.- The patient, a woman, aged 22 years, had lupus of both cheeks of 12 years' duration. The patches were covered with small superficial ulcers and the edges consisted of a zone of firm papules. These were healed after two months' treatment, though their outline still becomes red when the patient is excited.

CASE 5. -The patient was a woman, aged 35 years, with chronic pulmonary tuberculosis. Both apices were diseased and with cavity formation. Cough was troublesome, expectoration was moderate in quantity, and bacilli were numerous. With three months' injections the symptoms have greatly improved. The râles have quite disappeared from the left apex and nearly so from the right. The cough is very slight and only for a little while in the mornings. There is almost no expectoration and the bacilli have been absent for two months.

These cases, though not by any means conclusive, are at least encouraging and justify further investigation.

Glasgow.

\section{UPON AN ANTI-CHOLERA SERUM.}

BY ALLAN MACFADYEN, M.D. EDIN.,

FULIERIAN PROFESSOR OF PHYSIOLOGY, ROYAL INSTITUTION.

IT is generally accepted that the symptoms of cholera Asiatica are the result of an acute intoxication with certain products of the specific agent-the comma bacillus of Kocb. Further, that these symptoms are due not to a general invasion of the body by the bacillus but to an absorption of its toxins from the seat of infection-the intestine. An intoxication being the cardinal feature in the disease the character of the specific poison became naturally a subject of close investigation, and more than one attempt has been made to isolate it and to bring the treatment of cholera within the range of serum therapeutics. Little hope of success has been found to lie in the preparation and the use of a bactericidal serum. The serum therapy of cholera, if it is to meet with any success, must rest on an antitoxic basis, bearing in mind the clinical features of the disease. This also has generally been recognised by those who have endeavoured to introduce specific methods of treatment.

Numerous efforts have been made to obtain toxins from cultures of the cholera organism. Two theories, apparently contradictory, arose with regard to the nature of the specific toxins and gave rise to much controversy. It has been maintained, notably by French observers, that the poison is a genuine secretory product, both in vita and in vitro, and extracellular in the sense of being diffusible and soluble. Others, and pre-eminently German observers, maintain that the poison is inherent to the cell plasma and therefore of the intracellular type. The point of view determined in each case the method of investigation employed. The upholders of an extracellular poison endeavoured to obtain a secreted toxin by the cultivation of the bacillus on fluid laboratory soils, whilst those who beld the poison to be intracellular worked with the killed and undoubtedly toxic bodies of the bacilli. It may be useful to summarise the main results that were obtained inasmuch as a good deal of the work dates ten years back.
Among the earlier observers Petri ${ }^{1}$ grew the comma bacillus on peptone soils and obtained a heat-resistant proteid termed by him "toxo-peptone." The entire cultures, sterilised by heat, proved to be more toxic than their filtrates. Hueppe and Scholl ${ }^{2}$ cultivated the bacilli in fresh sterile eggø. The alcoholic precipitate from the egg yielded an aqueous extract poisonous to the guinea-pig. This effect was, however, shown to be due mainly to ordinary decomposition products and the retained alcohol. Gamaleia, as the result of his investigations, came to the conclusion that there are several cholera toxins. The organisms grown on a special veal broth yielded two soluble poisons - the one, thermolabile, produced diarrhœa in the rabbit, and the other, thermostable, caused an intoxication but without diarrhœa The doses required to bring about these effects were considerable. Behring and Ransom in $1895^{\text {described }}$ a soluble poison obtained by them from fluid cultures of the comma bacillus which was heat-resistant and acutely toxic to the guinea-pig in concentrated doses. For this poison they were able to prepare an antitoxin. It can hardly be supposed that these observers were dealing so muoh with the genuine poison as with secondary products which are to be met with in old cultures of the cholera and other organisms. Nor does their serum appear to have exhibited antitoxic properties much more striking than those of the normal serum. The important researches of Metchnikoff, Roux, and Salimbeni 5 call for detailed notice, both on account of the ingenious methods employed and the results obtained. Highly virulent cultures of the comma bacillus, prepared by cultivation in collodion sacs in the peritoneal carity of the guinea-pig, were sown on a special medium containing 2 per cent. peptone, 2 per cent. gelatin, and 1 per cent. sodic chloride. A short period of incubation was given to the cultures in order to obtain the toxin before it had undergone modification. The cultures reached their maximum toxicity on the fourth day and were then filtered. The filtrate was toxic on subcutaneous injection in doses of 0.3 cubic centimetre per 100 grammes weight of guinea-pig. This toxin, similarly to Behring and Ransom's, was not sensibly modified at boiling point but lost its activity under the action of air and light. The guinea-pig was most susceptible to the toxin, other animals, such as the rabbit, requiring larger doses. The poison acted subcutaneously and peritoneally. The effects consisted in hypothermia, peritonitis, fluid distension of the small intestine, along with hyperæmia of the abdominal organs. These results appear simple and conclusive in favour of the toxin obtained being a genuine secretion product of the bacilli. They are, however, open to another interpretationviz., that these observers were really dealing with toxic elements of the comma bacillus which had been released by autolysis and disintegration of the cells. The death-rate of organisms, even in a young cholera culture, is rapid. Gottschlich and Weigang ${ }^{6}$ found that in a two-days' culture of cholera at $37^{\circ} \mathrm{C}$. only about 10 per cent. of the bacilli were alive and on the third day at most 1 per cent. It still remains to me doubtful if such poisons are of the same character as those developed in the body in the course of a cholera infection.

Pfeiffer is the most distinguished upholder of the doctrine that the cholera poison is contained in the protoplasm of the cell. Young broth cultures have no marked toxic effect on filtration. On the other hand, carefully killed young agar cultures produce an acute intoxication of the same nature as that produced by living cultures. This has been demonstrated by Pfeiffer, in a most careful series of experiments. There can be little doubt that the primary cholera toxin is of the endocellular type, of an unstable character, and is easily converted into less toxic modifications. Its demonstration, therefore, requires a careful and conservative technique. Subsequent research has not substantially modified the respective standpoints indicated except in so far as Pfeiffer's conclusions have met with the widest acceptance. Since 1896 neither Behring and Ransom nor

1 Petri : Arbeiten aus dem Kaiserlichen Gesundheitsamte, Band vi., 1890.

Hueppe: Deutsche Medicinische Wochenschrift, p. 417, 1891

3 Gamaleia : Archives de Médecine Expérimentale, p. 172, 1892. 4 Behring: Deutsche Medicinisehe Wochenschrift, p. 294, 1898 ansom - ibid., p. 457, 1895 . 5 Metchnikoff, Roux, and Salimbeni : Annales de l'Institut Pasteur,
vol. x., p. 257, 1896 .

6 Gottschlich and Weigang: Zeitschrift für Hygiene, p. 376, vol. xx. 895.

7 Pfeiffer, ibid., vol. xi., 1892, p. 393; vol. xv., 1894, p. 268; and vol. xx., 1895, p. 217. 